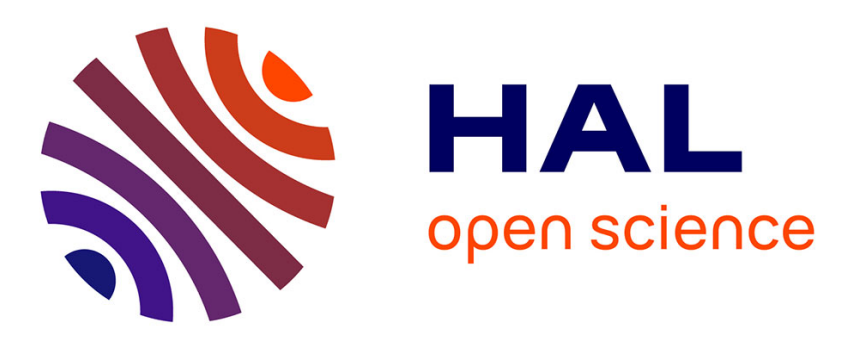

\title{
Graphene-based textured surface by pulsed laser deposition as a robust platform for surface enhanced Raman scattering applications
}

Teddy Tite, Christophe Donnet, A.-S. Loir, Stéphanie Reynaud, J.Y. Michalon, Francis Vocanson, Florence Garrelie

\section{To cite this version:}

Teddy Tite, Christophe Donnet, A.-S. Loir, Stéphanie Reynaud, J.Y. Michalon, et al.. Graphenebased textured surface by pulsed laser deposition as a robust platform for surface enhanced Raman scattering applications. Applied Physics Letters, 2014, pp.Volume 104, 041912. 10.1063/1.4863824 . ujm-00948962

\section{HAL Id: ujm-00948962}

\section{https://hal-ujm.archives-ouvertes.fr/ujm-00948962}

Submitted on 19 Feb 2014

HAL is a multi-disciplinary open access archive for the deposit and dissemination of scientific research documents, whether they are published or not. The documents may come from teaching and research institutions in France or abroad, or from public or private research centers.
L'archive ouverte pluridisciplinaire HAL, est destinée au dépôt et à la diffusion de documents scientifiques de niveau recherche, publiés ou non, émanant des établissements d'enseignement et de recherche français ou étrangers, des laboratoires publics ou privés. 


\title{
Graphene-based textured surface by pulsed laser deposition as a robust platform for surface enhanced Raman scattering applications
}

\author{
T. Tite, C. Donnet, A. -S. Loir, S. Reynaud, J. -Y. Michalon, F. Vocanson, and F. Garrelie* \\ Université de Lyon, F-42023, Saint-Etienne, France, CNRS, UMR5516, Laboratoire Hubert Curien, F-42000, Saint-Etienne, France, Université \\ de Saint-Etienne, Jean Monnet, F-42000, Saint-Etienne, France.E-mail: florence.garrelie@univ-st-etienne.fr
}

\begin{abstract}
We have developed a surface enhanced Raman scattering (SERS)-active substrate based on gold nanoparticles-decorated few-layer (fl) graphene grown by pulsed laser deposition. Diamond-Like Carbon film has been converted to fl-graphene after thermal annealing at low temperature. The formation of fl-graphene was confirmed by Raman spectroscopy, and surface morphology was highlighted by scanning electron microscopy. We found that textured fl-graphene film with nanoscale roughness was highly beneficial for SERS detection. Rhodamine $6 \mathrm{G}$ and $p$-aminothiophenol proposed as test molecules were detected with high sensitivity. The detection at low concentration of deltamethrin, an active molecule of a commercial pesticide was further demonstrated.
\end{abstract}

Since its discovery, graphene has attracted tremendous interest due to its exceptional physical and chemical properties. $^{1-2}$ Graphene has potential applications in many fields, including nanoelectronics, photonics, biochemistry, and energy storage. ${ }^{2}$ Despite its extraordinary properties, it is worth mentioning that pristine graphene is not very useful for practical applications because of its zero band gap, low carrier density and chemical inertness. ${ }^{3}$ Several routes have been explored in attempts to alter its surface and electronic structure, which include mechanical strain, ${ }^{3,4}$ patterning/texturing, ${ }^{5}$ and chemical functionalization through impurities, defects and adsorbates. ${ }^{6,7}$ Although defects are usually considered detrimental to the properties of graphene, it is worth to notice that they are opening many gateways for a wide range of new applications. ${ }^{3,7}$ Of our particular interest, decoration of graphene sheets with nanoparticles are new hybrids materials that can be used as catalysts, supercapacitors and biosensors. ${ }^{6}$ It was reported that graphene decorated $\mathrm{Au}$ or $\mathrm{Ag}$ nanoparticles (NPs) can effectively enhance Raman signals of absorbed organic molecules that makes it a useful surface-enhanced Raman scattering (SERS) substrate. ${ }^{8}$ Among the different methods used for fabricating graphene/AuNPs composites, the synthesis of reduced graphene oxide composites is the most popular one. ${ }^{8}$ Graphene oxide is usually preferred because of its rich chemical structure and the presence of defective active oxygen sites, which can enhance the graphene-metal/molecule binding. ${ }^{6}$ Although, new routes are under current investigations to provide better surface control synthesis and homogeneity, ${ }^{9,10}$ the preparation methods are still challenging. Recently, graphene films based on the conversion of solid carbon feedstock with metal catalyst film through thermal treatment have been obtained. Orofeo et al. indicated that the use of amorphous carbon (a-C) as solid source could provide an interesting approach to have a better control of the surface properties of graphene. ${ }^{11}$ Polycristalline $\mathrm{Co}$ and $\mathrm{Ni}$ thin films are generally used because of the high carbon solubility herein. ${ }^{11,12}$ However, although the synthesis of graphene from a-C thin film has become an attractive research activity, so far the applications are still unexplored.

Herein, we report the synthesis of few layer (fl) graphene by pulsed laser deposition (PLD) and investigate its applications as a SERS substrate for biomolecular detection. PLD has the advantages to be a conceptually simple, versatile, fast, costeffective and scalable technique to design amorphous carbon such as Diamond-Like-Carbon (DLC) thin films. Moreover, by providing energetic carbon species, PLD is an emerging technique to growth graphene at low temperature. ${ }^{13,14} \mathrm{Au}$ NPs were deposited on the fl-graphene to check the SERS activity. Rhodamine $6 \mathrm{G}, p$-aminothiophenol and a commercial pesticide were detected with a very good sensitivity. It is demonstrated that graphene synthesized from amorphous carbon can serve as an alternative SERS platform for molecular biosensing.

A thin DLC film ( $20 \mathrm{~nm}$ thick) was grown on a n-doped $\mathrm{Si}$ substrate under high vacuum condition by ablating a graphite target (99.997\% purity) with an excimer laser in a deposition chamber evacuated to a base pressure of about $10^{-4} \mathrm{~Pa}$. A KrF laser with a wavelength of $248 \mathrm{~nm}$, a pulse duration of $20 \mathrm{~ns}$, a repetition rate of $10 \mathrm{~Hz}$ and an energy per pulse of $400 \mathrm{~mJ}$ was used for the ablation. The energy density of the pulsed laser was set to $15 \mathrm{~J} \mathrm{~cm}^{-2}$ and the deposition rate of carbon was $2 \mathrm{~nm} \mathrm{~min}{ }^{-1}$. Subsequently, a thin nickel film (99.99\% purity, 150 $\mathrm{nm}$ thick) was deposited by thermal evaporation on top of the DLC film. The samples were further annealed $\left(780^{\circ} \mathrm{C}\right.$ during 45 min) in vacuum and were cooled down naturally to room temperature. Unpolarized micro-Raman experiments were carried out at room temperature with a multi-wavelength spectrometer to check the formation as well as the quality of graphene thin film. ${ }^{15}$ The spectral resolution was $\sim 2 \mathrm{~cm}^{-1}$. The laser beam was focused on the sample with $\times 100$ objective and the laser power was kept at $3 \mathrm{~mW}$ or below $0.2 \mathrm{~mW}$ for SERS detection to avoid any deterioration of the materials. The scattered Raman signal was then measured with an Aramis Jobin Yvon confocal spectrometer equipped with a chargecoupled device camera. Scanning electron microscopy (SEMFEG) (FEI, NovaNanoSEM) has been also used to characterize the films. To test for SERS activity, gold nanoparticles (Au NPs) were prepared following a similar procedure reported in the literature. ${ }^{16}$

Figure 1(a) shows the Raman spectra at $442 \mathrm{~nm}$ of a$\mathrm{C}(5 \mathrm{~nm}) / \mathrm{Si}$ before (spectrum i) and after thermal annealing (spectrum ii). The as-grown a-C thin film shows a broad 
assymetric Raman lineshape which was deconvoluted in two main Raman bands centered at $\sim 1385 \mathrm{~cm}^{-1}$ (D-peak) using a Lorentzian function and $\sim 1530 \mathrm{~cm}^{-1}$ (G-peak) using BreitWigner-Fano (BWF) function. ${ }^{17}$ The $\mathrm{sp}^{3}$ content in the film was estimated ca. $55-65 \%$ on the basis of the relationship between the $\mathrm{G}$ peak dispersion with laser wavelength and the degree of disorder. ${ }^{17}$ Although the thermal annealing induced a significant reduction of the $\mathrm{sp}^{3}$ content in the as-grown film in agreement with the formation of nano-sized graphene, ${ }^{18}$ we found that it is not enough to produce high quality graphene film in regards to the poor intensity of the $2 \mathrm{D}\left(\sim 2725 \mathrm{~cm}^{-1}\right)$ mode. In contrast, using Ni as catalyst, a sharp and intense symmetrical 2D peak could be obtained. Figure 1(b) shows a typical Raman spectrum at $442 \mathrm{~nm}$ of a sample Ni/a-C $(20 \mathrm{~nm}) / \mathrm{Si}$ after thermal annealing. The D, G and 2D peaks were observed at $\sim 1378,1590$ and 2752 $\mathrm{cm}^{-1}$. No shoulder was observed on the low frequency side of the $2 \mathrm{D}$ peak, suggesting that the stacking between the layers in not $\mathrm{AB}$ stacking, and the interlayer coupling is rather weak. ${ }^{19}$ The intensity ratio of $2 \mathrm{D} / \mathrm{G}$ is $\sim 0.4$ which indicates the formation of few layer (fl) graphene. ${ }^{1,12,14}$ It is worthwhile to notice an improvement of the results compared with those previously obtained by PLD, ${ }^{14}$ and moreover a comparable intensity ratio $2 \mathrm{D} / \mathrm{G}$ with those obtained by filtered cathodic vacuum arc technique, ${ }^{16}$ and standard reduced graphene oxide. ${ }^{20,21}$ The mechanism of formation of graphene on nickel generally involves (i) dissociation of solid carbon thin film, (ii) dissolution and diffusion of carbon in the metal during heating at high temperature, and (iii) segregation and/or precipitation of $\mathrm{C}$ atoms at the surface of the metal either at the growth temperature when the solid solution has been saturated, or during cooling because of the decrease of carbon solubility in the metal. ${ }^{22}$

SEM analysis indicates the formation of distinct surface morphologies after the sample $\mathrm{Ni} / \mathrm{a}-\mathrm{C}(20 \mathrm{~nm}) / \mathrm{Si}$ has been thermal annealed (Fig. 2(a)). The texturing of the surface is explained through a diffusion mechanism of $\mathrm{Ni}$ atoms into the
Si substrate during the heating with the concomitant formation of transition metals silicides. ${ }^{23}$ Such dynamical processes during the thermal heating are responsible of the formation of rough surface with a high defect density (upper part of Fig. 2(a)). According to AFM measurements, surface roughness extend from 3 to $20 \mathrm{~nm}$, with neighboring small areas ranging up to $60-100 \mathrm{~nm}$. In rough regions the average distance between defects is estimated between $10-15 \mathrm{~nm}$, which corresponds to a defect density in the range $\sim 4 \times 10^{11}-10^{12} \mathrm{~cm}^{-2}{ }^{24}$ At nanoscale, defects and corrugated surface could be extremely useful since they could be exploited to tune the electronic and transport properties of graphene. ${ }^{5}$ In particular, it is worthwhile to notice that defects as chemically active sites and roughness as surface with higher specific area promote the adsorption and the detection of molecules. Recently, Xu et al. have fabricated a unique mechanically exfoliated graphene-veiled substrate, and have shown that both Au NPs and graphene topography should influence the SERS activity. ${ }^{25}$ In this context, the specific surface architecture of our material with large scale fl-graphene could be particularly attractive for the SERS which requires rough metallic surface for higher detection sensitivity.

In the present work, the NPs size deduced from transmission electron microscopy has been estimated at ca. $23 \mathrm{~nm}$ and by referring to ultraviolet-visible-near infrared absorption analysis, a sharp surface plasmon resonance (SPR) band of NPs was observed at $525 \mathrm{~nm}$ (not shown). To decorate the fl-graphene with Au NPs, a small droplet of AuNPs in colloidal solution was simply deposited onto the surface of the sample and rinsed in ultrapure water twice after being dried in air. Typical SEM image of the fl-graphene surface after Au NPs decoration is shown in Fig. 2(b). Here, we point out that these images have been taken after the sample has been unreasonably rinsed five times in ultrapure water. The AuNPs are obviously seen which indicates their good anchoring with the surface of the platform via electrostatic, Van der Waals and elastic interactions. In particular, surface roughness, topography and wettability may play a predominant role in the adhesion.

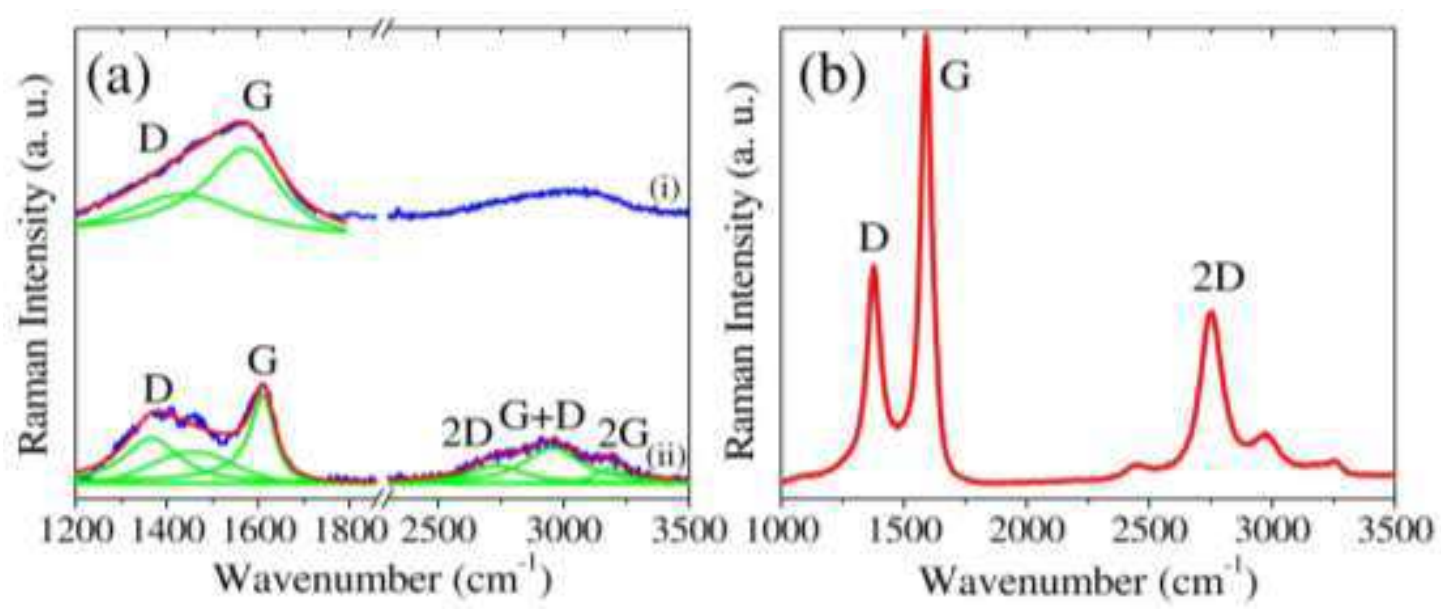

FIG. 1. Raman spectra at $442 \mathrm{~nm}$ of a-C(5nm)/Si (a) before (i), and after thermal processing (ii). Curves fitting are added in solid lines. Typical Raman spectrum at $442 \mathrm{~nm}$ of $\mathrm{Ni} / \mathrm{a}-\mathrm{C}(20 \mathrm{~nm}) / \mathrm{Si}$ after thermal annealing at $780^{\circ} \mathrm{C}(\mathrm{b})$. 


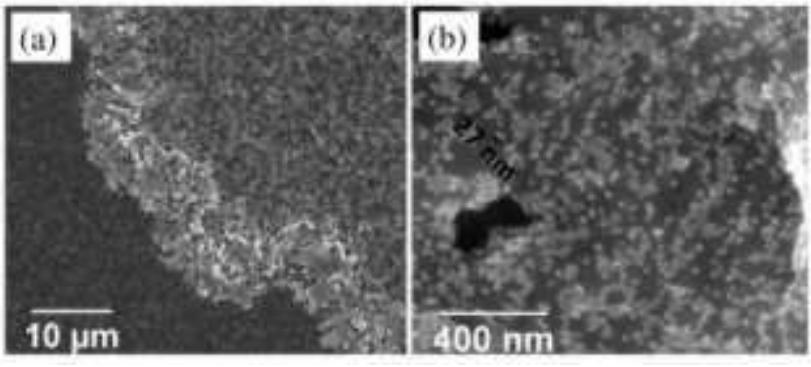

FIG. 2. Typical SEM images of $\mathrm{Ni} / \mathrm{a}-\mathrm{C}(20 \mathrm{~nm}) / \mathrm{Si}$ after thermal processing at $780^{\circ} \mathrm{C}$ before (a) and after Au NPs decoration (b).

To investigate whether the as-fabricated AuNPs-fl-graphene (Au-fl-G) substrates can be used as an effective SERS platform, rhodamine R6G and $p$-aminothiophenol ( $p$ ATP) were used as SERS probes because of their well-known vibrational properties. ${ }^{26,27}$ In recent papers, Yao et $a l .{ }^{10}$ and Huang et $a l .{ }^{9}$ have detected respectively, R6G and $p$ ATP by SERS using reduced graphene oxide as a platform, with a good sensitivity at the corresponding concentration $2 \times 10^{-5} \mathrm{M}$ and $10^{-3} \mathrm{M}$. Firstly, SERS activity was investigated by depositing a droplet of R6G diluted in ultrapure water with a concentration of $10^{-6} \mathrm{M}$ onto the surface of the substrate. Typical Raman spectra with and without R6G are shown in Fig. 3(a). The Raman intensities of D-band and G-band on the Au-fl-G/Si are stronger than that on fl-G/Si substrate, which was attributed to the coupled SPR absorption of AuNPs. ${ }^{28}$ Additional vibrational bands have been clearly observed after the deposition of a droplet of R6G on the surface of the sample Au-fl-G/Si, which has been rinsed twice in ultrapure water to remove the unbounded molecules (Fig. $3(\mathrm{a})$ ). These spectral features observed at $612,772,1186,1310$, $1361,1508,1575,1597$, and $1647 \mathrm{~cm}^{-1}\left( \pm 2 \mathrm{~cm}^{-1}\right)$ are due to vibration modes of the R6G molecule. ${ }^{26}$ In particular, we noticed that the intensity of the modes at 1647 and $1575 \mathrm{~cm}^{-1}$, which are attributed to $\mathrm{C}=\mathrm{C}$ symmetric stretching motions, is enhanced in comparison to others modes under a $488 \mathrm{~nm}$ excitation (Fig. 3(a)). This behavior was attributed to a change in their molecular geometries under resonant Raman excitation. ${ }^{26}$ Figure 3(b) shows a typical Raman mapping at 633 $\mathrm{nm}$ excitation of the intensity of the mode at $1647 \mathrm{~cm}^{-1}$ of R6G $\left(10^{-6} \mathrm{M}\right)$ taken in the rough region. Although the mode at 1647 $\mathrm{cm}^{-1}$ was chosen to evaluate the Raman enhancement magnitude, we argued that similar results were obtained for others modes. The colorful Raman cartography suggested rather a good and uniform detection of R6G. The SERS activity on the samples is interpreted as a combination between the electromagnetic (EM) and chemical (CM) mechanisms, ${ }^{29}$ although according our experimental results EM effects which are particularly sensitive to the inter-particles distance should prevailed. ${ }^{9}$ We emphasized that using our SERS platform, a sensitive and fast detection were obtained in regards to the low laser power density used $\left(6 \times 10^{2} \mathrm{~W} / \mathrm{cm}^{2}\right)$ and short acquisition time (6s). Having established that the as-fabricated fl-graphene sample is a SERS-active substrate, we employed it for the detection of $p$-aminothiophenol ( $p$ ATP) and a pesticide.

Various concentrations from $10^{-6} \mathrm{M}$ to $10^{-3} \mathrm{M}$ of $p$ ATP in methanol was dropped onto the SERS substrate, and rinsed twice in ultrapure water after the methanol was evaporated for SERS measurement. Figure 4(a) shows some typical Raman spectra of $p$ ATP on Au-fl-G/Si obtained at $633 \mathrm{~nm}$ excitation for the concentrations from $10^{-6} \mathrm{M}$ to $10^{-3} \mathrm{M}$. Two main additional peaks were observed at $\sim 1072$ and $1581 \mathrm{~cm}^{-1}$ in the Raman spectra after $1 \mu \mathrm{M}$ of $p$ ATP was adsorbed on the surface of the sample. The vibrational bands of $p$ ATP are further revealed when its concentration was increased from $10^{-6} \mathrm{M}$ to $10^{-3} \mathrm{M}$. At $10^{-3} \mathrm{M}$, the peaks of $p$ ATP were located at 1005 , $1079,1140,1177,1388,1432$, and $1588 \mathrm{~cm}^{-1}\left( \pm 2 \mathrm{~cm}^{-1}\right)$, in good agreement with previous results. ${ }^{12,32}$ The main peaks at 1079 and $1588 \mathrm{~cm}^{-1}$ are attributed to the vibration modes of $v_{\mathrm{C}-\mathrm{C}}+v_{\mathrm{C}-\mathrm{S}}$ and $v_{\mathrm{C}-\mathrm{C}}+\delta_{\mathrm{NH} 2}$, respectively. ${ }^{12,32}$ Compared with the spectra of $p$ ATP at $10^{-6} \mathrm{M}$, the main peaks of $p$ ATP at $10^{-3} \mathrm{M}$ are found blue shifted $(+0.9 \mathrm{meV})$, likely due to the interaction between $p$ ATP and the SERS substrate via phenyl-sulfur group.
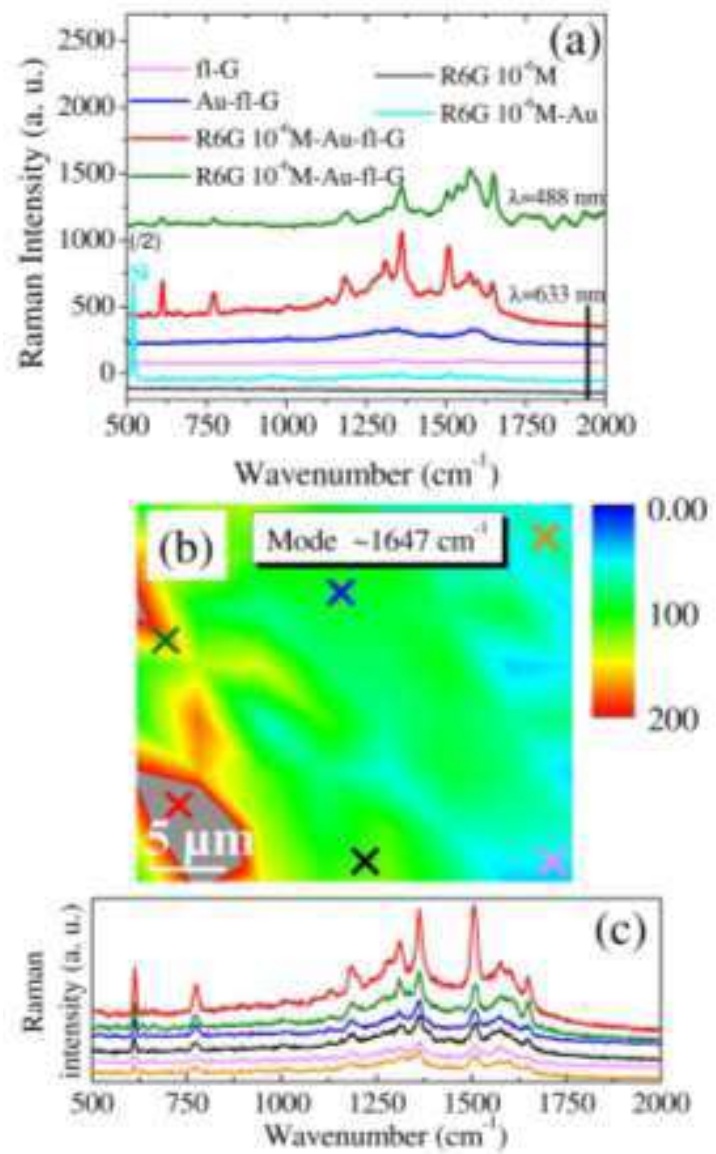

FIG.3. Raman spectra of R6G $\left(10^{-6} \mathrm{M}\right)$, R6G(10 $\left(10^{-6} \mathrm{M}\right)-\mathrm{Au} / \mathrm{Si}$, fl-graphene, Au-fl-G, and $\mathrm{R} 6 \mathrm{G}\left(10^{-6} \mathrm{M}\right)-\mathrm{Au}-\mathrm{fl}-\mathrm{G}$ at $633 \mathrm{~nm}$ and $488 \mathrm{~nm}$ wavelengths (a); typical Raman mapping at $633 \mathrm{~nm}$ of R6G $\left(10^{-6} \mathrm{M}\right)$ dropped on Au-fl-G (b); typical SERS spectra from the spots marked with corresponding colored cross in (b) are shown in (c).

The toxicity posed by pesticides even at very low concentrations has become a large concern for both health and environmental safety. Among all challenging methods developed for detection of contaminants, graphene is become a new playground due to its high adsorption efficiency. ${ }^{30}$ In this context, we propose to evaluate the SERS performance of the as-fabricated samples to detect deltamethrin, the active substance of a commercial agricultural pesticide Decis Protech. A study of cultured human keratinocyte cells exposed to deltamethrin has evaluated a cytotoxic dose between $10^{-4} \mathrm{M}$ and $2.5 \times 10^{-4} \mathrm{M} .{ }^{31}$ Fig. 4(b) shows the Raman spectra of deltamethrin on $\mathrm{Au}-\mathrm{fl}-\mathrm{G} / \mathrm{Si}$ with concentrations in deltamethrin increasing 
from $10^{-6} \mathrm{M}$ to $10^{-3} \mathrm{M}$. The intensity of Raman peaks of deltamethrin located at around 1001, 1456, 2880, 2935 and $3064 \mathrm{~cm}^{-1}$ increases with the deltamethrin concentration. It should be notice that the peak centered at $2140 \mathrm{~cm}^{-1}$ can be assigned to either polyyne chains $(-\mathrm{C} \equiv \mathrm{C}-)_{\mathrm{n}}$ with $\mathrm{n}=3-4$, or adsorbed $\mathrm{CO} .{ }^{32}$ It is interesting to point out that although the signal-to-noise and fluorescence background become worst by increasing the concentration of this pesticide, deltamethrin can still be detected below the cytotoxic dose range, which is important for environmental safety. Further investigation should be done to understand the sensitivity of deltamethrin to its environment, ${ }^{33}$ and its interaction with Au NPs decorated SERS platform.
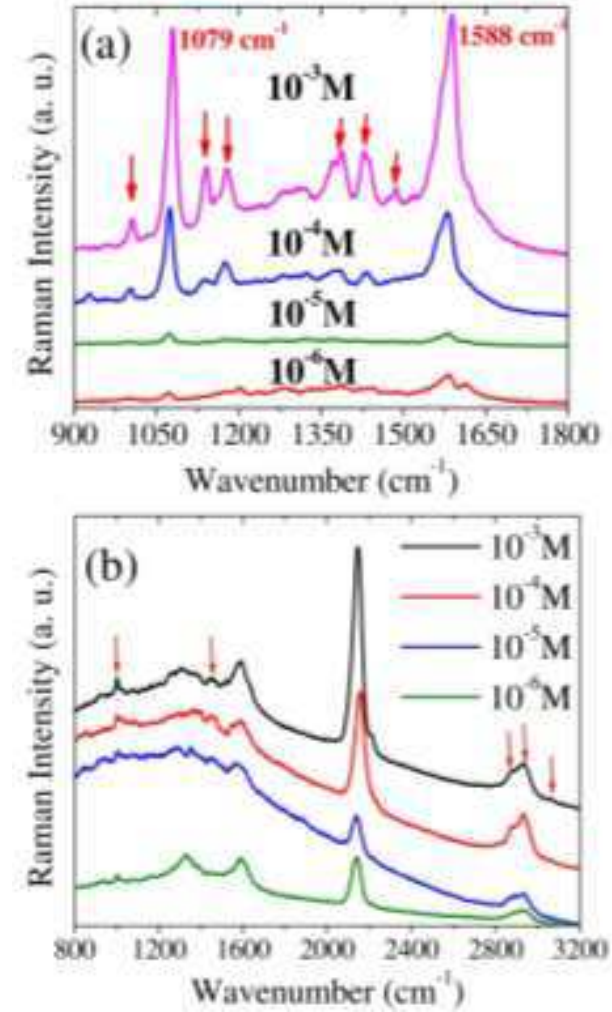

FIG.4. Raman spectra at $633 \mathrm{~nm}$ of $p$-aminothiophenol (pATP) (a) and deltamethrin (b) deposited on Au-fl-G at different concentrations (from $10^{-6} \mathrm{M}$ to $10^{-3} \mathrm{M}$ ). The arrows indicate the peaks position of $p$ ATP and deltamethrin.

In conclusion, we have shown the application of few layer (fl) graphene synthesized from solid state source as a highefficiency SERS-active substrate for molecular diagnostics. The method developed based on the conversion of PLD-grown amorphous carbon to large scale fl-graphene is simple, convenient, fast and cost effective. From the unique surface architecture of the as-fabricated fl-graphene with nanoscale roughness on Si substrate, rhodamine, p-aminothiophenol and a commercial pesticide have been detected at low concentration with high sensitivity. We expect that the Au NPs decorated fl-graphene substrate will become a practical and powerful platform for the harvesting of SERS signal of various molecules, such as pesticides or pollutants for environmental safety.

The authors would like to acknowledge M. Nicolas Boyer from the laboratory BVPAM Saint-Etienne for the preparation of the pesticide solution.
${ }^{1}$ C. Yan, J. H. Cho, and J. -H. Ahn, Nanoscale 4, 4870 (2012).

${ }^{2}$ K. S. Novoselov, V. I. Fal'ko, L. Colombo, P. R. Gellert, M. G. Schwab, and K. Kim, Nature, 490, 192 (2012).

${ }^{3}$ M. A. Bissett, M. Tsuji, and H. Ago, J. Phys. Chem. C 117, 3152 (2013).

${ }^{4}$ Z. H. Ni, T. Yu,Y. H. Lu, Y. Y. Wang, Y. P. Feng, and Z. X. Shen, ACS Nano 2, 2301(2008).

${ }^{5}$ J. Feng, W. Li, X. Qian, J. Qi, L. Qi, and J. Li, Nanoscale 4, 4883 (2012).

${ }^{6}$ V. Georgakilas, M. Otyepka, A. B. Bourlinos, V. Chandra, N. Kim, K. Christian Kemp, P. Hobza, R. Zboril, and K. S. Kim, Chem. Rev. 112, 6156 (2012).

${ }^{7}$ S. T. Pantelides, Y. Puzyrev, L. Tsetseris, and B. Wang, MRS bulletin 37, 1187 (2012)

${ }^{8}$ W. Xu, N. Mao, and J. Zhang, Small 9, 8, 1206 (2013).

${ }^{9}$ J. Huang, L. Zhang, B. Chen, N. Ji, F. Chen, Y. Zhang, and Z. Zhang, Nanoscale 2, 2733 (2010)

${ }^{10}$ H. Yao, L. Jin, H-J. Sue, Y. Sumi, and R. Nishimura, J. Mater. Chem. A 1, 10783 (2013).

${ }^{11}$ C. M. Orofeo, H. Ago, B. Hu, and M. Tsuji, Nano Res. 4, 531 (2011).

${ }^{12}$ O. S. Panwar, A. K. Kesarwani, S. Rangnath Dhakate, B. P. Singh, R.

Kumar Rakshit, A. Bisht, and S. Chockalingam, J. Vac. Sci. Technol. B 31, 040602 (2013).

${ }^{13}$ S. R. Sarath Kumar, and H. N. Alshareef, Appl. Phys. Lett. 102, 012110 (2013).

${ }^{14}$ A. T. T. Koh, Y. M. Foong, and D. H. C. Chua, Diamond and Related Materials 25, 98 (2012).

${ }^{15}$ L. M. Malard, M. A. Pimenta, G. Dresselhaus, and M. S. Dresselhaus, Physics Reports 473, 51 (2009).

${ }^{16} \mathrm{G}$. Frens, Nature 241, 20 (1973).

${ }^{17}$ A. C. Ferrari, and J. Robertson, Phys. Rev. B 64, 075414 (2001).

${ }^{18}$ E. Cappelli, S. Orlando, V. Morandi, M. Servidori, and C. Scilletta, Journal of Physics: Conference Series 59, 616 (2007).

${ }^{19}$ A. C. Ferrari, J. C. Meyer, V. Scardaci, C. Casiraghi, M. Lazzeri, F. Mauri, S. Piscanec, D. Jiang, K. S. Novoselov, S. Roth, and A. K. Geim, Phys. Rev. Lett. 97, 187401 (2006).

${ }^{20}$ X. Li, B. K. Tay, J. Li, D. Tan, C. W. Tan, and K. Liang, Nanoscale Research Letters 7, 205 (2012).

${ }^{21}$ C. Petridis, Y.-H. Lin, K. Savva, G. Eda, E. Kymakis, T. D. Anthopoulos, and E. Stratakis, Appl. Phys. Lett. 102, 093115 (2013).

${ }^{22}$ X. S. Li, W. W. Cai, L. Colombo, and R. S. Ruoff, Nano Lett. 9, 4268 (2009).

${ }^{23}$ M. Bhaskaran, S. Sriram, T.S. Perova, V. Ermakov, G.J. Thorogood, K.T. Short, A.S. Holland, Micron 40, 89 (2009).

${ }^{24}$ L. G. Cançado, K. Takai, T. Enoki, M. Endo, Y. A. Kim, H. Mizusaki, A. Jorio, L. N. Coelho, R. Magalhães-Paniago, and M. A. Pimenta, Appl. Phys. Lett. 88, 163106, (2006).

${ }^{25}$ W. Xu , J. Xiao, Y. Chen, Y. Chen , X. Ling, and J. Zhang, Adv. Mater. 25, 928 (2013).

${ }^{26} \mathrm{H}$. Watanabe, N. Hayazawa, Y. Inouye, and S. Kawata, J. Phys. Chem. B 109, 5012 (2005).

${ }^{27}$ J. Ye, J. A. Hutchison, H. Uji-i, J. Hofkens, L. Lagae, G. Maes, G. Borghs, and P. V. Dorpe, Nanoscale 4, 1606 (2012).

${ }^{28}$ A. N. Sidorov, G. W. Sławiński, A. H. Jayatissa, F. P. Zamborini, and G. U. Sumanasekera, Carbon 50, 699 (2012).

${ }^{29}$ E. C. Le Ru, and P. G. Etchegoin, , MRS bulletin 38, 631 (2013).

${ }^{30}$ S. M. Maliyekkal, T. S. Sreeprasad, D. Krishnan, S. Kouser, A. K Mishra, U. V. Waghmare, and T. Pradeep, Small 9, 2, 273 (2013).

${ }^{31}$ G. Perna, M. Lasalvia, P. D'Antonio, G. Quartucci, and V. Capozzi, Vibrational Spectroscopy 57, 1, 55 (2011).

${ }^{32}$ A. Hu, Q.-B. Lu, W. W. Duley, and M. Rybachuk, The Journal of Chemical Physics 126, 154705 (2007).

${ }^{33}$ H. M. Coleman, T. Trinh, N. Le-Minh, M. Klein, D. J. Roser, R. W. Tucker, R. M. Stuetz, G. Peters, and S. J. Khan, Environmental Pollution 174, 265 (2013). 